\title{
Zooming in on Ultracold Matter
}

\begin{abstract}
Two superresolution microscopy methods can image the atomic density of ultracold quantum gases with nanometer resolution.
\end{abstract}

\section{by Stefan Kuhr*}

U ltracold atoms are an exceptionally versatile platform to test novel physical concepts. They have greatly advanced our understanding of the physics of many-body systems and allowed precision measurements of fundamental constants. They are also a promising architecture for quantum computation and quantum simulation. A key to the practicality of ultracold atoms is the ability to image them with high spatial resolution. Available microscopy schemes have reached sufficient resolution to detect individual atoms trapped in optical lattices with submicron spacings, but their spatial resolution is typically limited to about half the wavelength of the imaging light. Now, two independent teams-one led by Cheng Chin from the University of Chicago, Illinois [1], and the other led by Steve Rolston and Trey Porto from the University of Maryland, College Park [2]—have reported

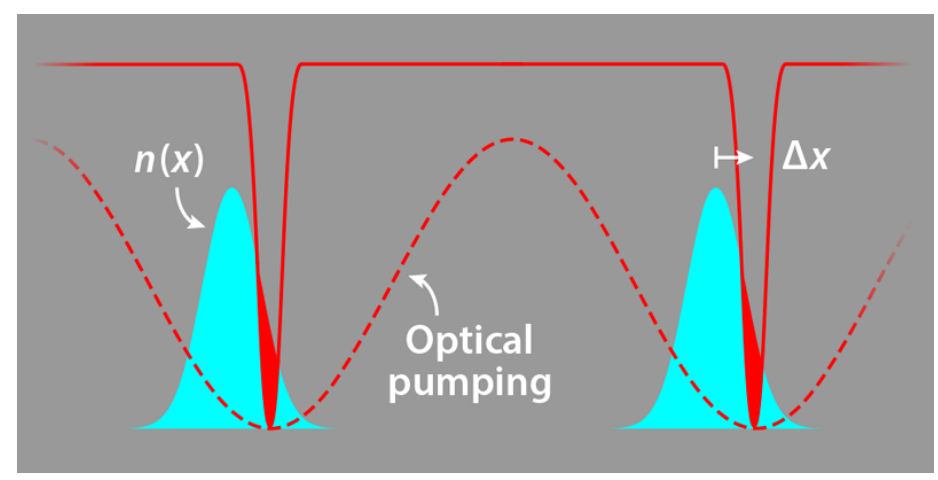

Figure 1: Sketch of the superresolution imaging scheme demonstrated by Chin's team [1]. Cesium atoms (blue shaded areas) are trapped at the sites of an optical lattice. An overlapping standing wave created by lasers (dashed line), displaced by $\Delta x$ with respect to the trapping lattice, excites most atoms within each lattice site, while leaving a narrow window in which the fraction of excited atoms is zero. By scanning the window, the setup can map the atomic density distribution $n(x)$ with subwavelength resolution. (APS/Alan Stonebraker)

\footnotetext{
*Department of Physics, University of Strathclyde, Glasgow, United Kingdom
}

subwavelength-resolution imaging techniques for ultracold atoms. The methods, capable of resolving objects up to 50 times smaller than the optical wavelength, have allowed the teams to map the shape of atomic density distributions on nanometer scales. Nanoscale maps of atomic density will be important observables for probing many-body effects in cold atomic and molecular systems.

Researchers have devised several methods to image cold atomic gases. The most popular one involves switching off the trapping lattices and imaging the atomic absorption after the atoms have expanded. Another recent technique, dubbed quantum-gas microscopy, can resolve single atoms within the optical lattice [3-5]. Relying on standard optical imaging, these methods are fundamentally limited by diffraction. As Ernst Abbe found in 1873, the minimum resolvable distance between two spots is proportional to the wavelength of the imaging light. For imaging at optical wavelengths, the resolution typically lies between 0.3 and $0.7 \mu \mathrm{m}$. However, many interesting features in the atomic distribution (represented by the atomic density wave function) are often on the scale of nanometers. Several approaches have attempted to overcome the diffraction limit. One group achieved a resolution of $150 \mathrm{~nm}$ by probing ultracold atoms with the focused electron beam of a scanning electron microscope [6], but such a microscope cannot be easily integrated in cold-atom setups. Other researchers have implemented a combination of laser light and microwave fields to manipulate individual atoms with subwavelength resolution [7], but the technique wouldn't be suitable for mapping atomic density wave functions.

The teams of Chin and of Rolston and Porto have developed new imaging techniques for ultracold atoms that significantly surpass Abbe's limit. Their work builds on developments in superresolution microscopy, which were recognized by the 2014 Nobel Prize in Chemistry. In particular, the teams' superresolution strategies are reminiscent of that used in "stimulated emission depletion microscopy," in which the nonlinear response of fluorescent markers is exploited to localize individual markers with nanometer resolution [8]. A similar nonlinear response of atoms is put to use by the two teams. This approach relies on preparing only a fraction of the atoms in a specific atomic energy state. This "select" population is confined to an extremely small spatial window (Fig. 1), which, thanks to saturation effects, can be much smaller than the optical wavelength. By scanning the 
position of the window over the cloud and selectively imaging only the atoms inside the window, the spatial structure of the cloud can be mapped with nanometer resolution.

The group from the University of Chicago used cold cesium atoms trapped in a one-dimensional optical lattice with a periodicity of $426 \mathrm{~nm}$, created by a standing-wave pattern from an infrared laser. With a laser at a different wavelength, the researchers generated another standing wave that had a similar periodicity but could be shifted along the lattice direction with nanometer precision. The light from this second laser drove a specific transition between two hyperfine states of the atoms. Since just a few photons are sufficient to cause this transition, only atoms within a narrow window around the nodes of the second lattice remained unexcited. By scanning the position of this unexcited window across the lattice and imaging the fraction of atoms in the excited state, the team could build a map of the atomic density distribution with a resolution of just over $30 \mathrm{~nm}$, determined by the width of the window. To demonstrate the capabilities of their microscopy technique, the researchers quickly displaced the trapping lattice by only a fraction of the wavelength and then imaged the evolution of the atomic density distribution as a function of time. They could see that the displacement induced an oscillatory motion of the atomic wave packets with an amplitude of about $100 \mathrm{~nm}$.

The team from the University of Maryland followed a similar approach, using ytterbium atoms in a one-dimensional lattice. Instead of leaving the atoms unexcited in a narrow window, as in the Chicago team's setup, their scheme only excited atoms within the window. The authors applied a combination of a standing-wave laser field and a homogeneous field. The two fields transfered the atoms into a so-called dark state made up of the quantum superposition of two internal energy states. As a result of the spatial distribution of the laser fields, the exact makeup of this superposition varied across the atomic cloud. At certain positions, corresponding to the nodes of the control field, the fields produced a subwavelength-sized window in which the atoms were predominantly in a specific dark state superposition. By moving the window's position and selectively detecting the atoms in that state, the researchers acquired maps of the atomic density with an impressive resolution of $11 \mathrm{~nm}$, about one fiftieth of the wavelength of the experimental lasers. The team demonstrated the power of the technique by imaging the atomic wave functions for two different optical lattices, one with a sinusoidal shape and the other with an approximately rectangular shape (a Kronig-Penney lattice). The setup could distinguish shape differences between the wave functions for the two cases on scales of tens of nanometers.
One can anticipate that new superresolution imaging of ultracold atoms will benefit a wide array of experiments relying on the direct measurement of atomic density wave functions, and of their dynamics, in many-body quantum systems. The technique could, for instance, image vortices with submicron sizes that occur in stirred Bose-Einstein condensates and other trapped configurations that are not periodic [9]. It could also provide a detailed picture of the complex wave functions of atoms in optical lattices in which the atoms occupy high-energy bands. Such lattices show promise as quantum emulators of exotic solid-state crystals [10]. Finally, it could allow subwavelength-resolution nondemolition measurements of the density of atoms inside an optical cavity-a setup that could see the quantum motion of atoms without disturbing them [11].

This research is published in Physical Review X.

\section{REFERENCES}

[1] M. McDonald, J. Trisnadi, K.-X. Yao, and C. Chin, "Superresolution microscopy of cold atoms in an optical lattice," Phys. Rev. X 9, 021001 (2019).

[2] S. Subhankar, Y. Wang, T.-C. Tsui, S. L. Rolston, and J. V. Porto, "Nanoscale atomic density microscopy," Phys. Rev. X 9, 021002 (2019).

[3] W. S. Bakr, J. I. Gillen, A. Peng, S. Fölling, and M. Greiner, "A quantum gas microscope for detecting single atoms in a Hubbard-regime optical lattice," Nature 462, 74 (2009).

[4] S. Kuhr, "Quantum-gas microscopes: A new tool for cold-atom quantum simulators," Natl. Sci. Rev. 3, 170 (2016).

[5] C. Gross and I. Bloch, "Quantum simulations with ultracold atoms in optical lattices," Science 357, 995 (2017).

[6] T. Gericke, P. Würtz, D. Reitz, T. Langen, and H. Ott, "Highresolution scanning electron microscopy of an ultracold quantum gas," Nat. Phys. 4, 949 (2008).

[7] C. Weitenberg, M. Endres, J. F. Sherson, M. Cheneau, P. Schauß, T. Fukuhara, I. Bloch, and S. Kuhr, "Single-spin addressing in an atomic Mott insulator," Nature 471, 319 (2011).

[8] S. W. Hell and J. Wichmann, "Breaking the diffraction resolution limit by stimulated emission: Stimulated-emissiondepletion fluorescence microscopy," Opt. Lett. 19, 780 (1994).

[9] K. W. Madison, F. Chevy, W. Wohlleben, and J. Dalibard, "Vortex formation in a stirred Bose-Einstein condensate," Phys. Rev. Lett. 84, 806 (2000).

[10] X. Li and W. V. Liu, "Physics of higher orbital bands in optical lattices: A review,” Rep. Prog. Phys. 79, 116401 (2016).

[11] D. Yang, C. Laflamme, D. V. Vasilyev, M. A. Baranov, and P. Zoller, "Theory of a quantum scanning microscope for cold atoms," Phys. Rev. Lett. 120, 133601 (2018).

10.1103/Physics.12.36 